\title{
Histopathological and immunohistochemical characterisation of hepatic granulomas in Leishmania donovani-infected BALB/c mice: a time-course study
}

\author{
Francisco J. Salguero', Waldo L. Garcia-Jimenez ${ }^{1}$, Isadora Lima ${ }^{1,2}$ and Karin Seifert ${ }^{3^{*}}$
}

\begin{abstract}
Background: Visceral leishmaniasis (VL) is a neglected tropical disease (NTD), caused by the intracellular protozoan parasites Leishmania donovani and Leishmania infantum. Symptomatic VL is considered fatal when left untreated. At present, there is no effective vaccine licensed for human use and available chemotherapies have limitations. Understanding the local immune mechanisms required for the control of infection is a key factor for developing effective vaccines and therapeutics.

Methods: We have investigated the development of the typical granulomatous lesions in the liver in experimental $V L$ over time, together with the local immune responses. BALB/C mice were infected intravenously with a dose of $2 \times 10^{7} \mathrm{~L}$. donovani amastigotes (MHOM/ET/67/HU3) and sacrificed at 15, 35 and 63 days post-infection (dpi). Histopathology and immunohistochemical techniques were used for the detection of Leishmania antigen, selected cell types including B and T lymphocytes, macrophages and neutrophils (CD45R-B220+, CD3+, F4/80+ and Ly-6G+) and iNOS.

Results: Granulomatous lesions were identified as early as $15 \mathrm{dpi}$ in the livers of all infected animals. Three categories were used to classify liver granulomas (immature, mature and clear). Clear granulomas were exclusively detected from 35 dpi onwards. Kupffer cells (F4/80+) were predominant in immature granulomas, regardless of the dpi. Nonetheless, the highest expression was found $63 \mathrm{dpi}$. Positive staining for iNOS was mainly observed in the cytoplasm of fused Kupffer cells and the highest expression observed at 35 dpi. T cells (CD3+) and B cells (CD45R-B220+) were predominant in more advanced granuloma stages, probably related to the establishment of acquired immunity. Neutrophils (Ly-6G+) were predominantly observed in mature granulomas with the highest expression at $15 \mathrm{dpi}$. Neutrophils were lower in numbers compared to other cell types, particularly at later time points.

Conclusions: Our results reflect the role of macrophages during the early stage of infection and the establishment of a lymphocytic response to control the infection in more advanced stages.
\end{abstract}

Keywords: Leishmania donovani, Visceral leishmaniasis, Histopathology, Immunohistochemistry, Macrophage, Granuloma

\footnotetext{
* Correspondence: karin.seifert@lshtm.ac.uk

${ }^{3}$ Faculty of Infectious and Tropical Diseases, London School of Hygiene \&

Tropical Medicine, London, UK

Full list of author information is available at the end of the article
} 


\section{Background}

Leishmaniasis is one of the most prevalent parasitic public health problems worldwide $[1,2]$. This term includes cutaneous leishmaniasis (CL), mucocutaneous leishmaniasis [3] and visceral leishmaniasis (VL) [2, 4]. $\mathrm{VL}$ is caused by the intracellular protozoan parasites Leishmania donovani in Asia and Africa and Leishmania infantum in Latin America and the Mediterranean region [5]. VL has a high mortality rate if untreated and is estimated to cause $0.2-0.4$ million new cases and 20,000-40,000 deaths per year worldwide [1]. Parasites are transmitted by female phlebotomine sandflies to mammalian hosts. Humans are the only known reservoir of $L$. donovani, while canines are the main reservoir for L. infantum [6]. In the Mediterranean basin and Latin America, VL is considered a zoonosis caused by $L$. infantum. Northward spread of VL endemic foci in Italy has been reported [7].

At present, there is no vaccine licensed for human use against VL. Limitations of current chemotherapeutic treatments include drug toxicity, long treatment courses, challenging routes of drug administration, drug stability in hot climates and geographical differences in clinical responses to treatment $[8,9]$.

Understanding the immune mechanisms required for control of infection within the varied tissue microenvironments that contain Leishmania-infected macrophages is a key factor for developing effective vaccines and therapeutics. Due to the intrusive techniques required to analyse such responses in human VL patients, current knowledge of host responses in tissues largely derives from experimental animal models, which include mice, hamsters and dogs. The granulomatous pathology of leishmaniasis, across different disease manifestations and in humans and animal hosts, has recently been reviewed [10]. Many of the cellular and molecular components of acquired immunity necessary for the formation, maintenance and effector function of granulomas have been characterised through the use of genetargeted mice or in vivo administration of neutralizing or depleting monoclonal antibodies [10-12]. Here we characterized the local host and immune response in hepatic tissue from $L$. donovani-infected BALB/c mice over time, using histopathological and immunohistochemical analyses.

\section{Methods}

\section{Experimental design}

A total of $24 \mathrm{BALB} / \mathrm{c}$ mice (Charles River, UK) were used for this study. Leishmania donovani (MHOM/ET/ $67 /$ HU3) amastigotes were harvested from the spleen of a Rag-1-knockout (B6) mouse (LSHTM breeding colony, infected > 40 days), re-suspended in RPMI 1640 medium without serum and used for the infection of 18 mice through a $0.2 \mathrm{ml}$ intravenous bolus injection into a tail vein, corresponding to $2 \times 10^{7}$ amastigotes. One group of 6 mice was left uninfected.

Groups of 6 mice were euthanized by cervical dislocation at three different time points, $15 \mathrm{dpi}$ (6 infected mice +6 uninfected mice), 35 dpi (6 infected mice) and 63 dpi (6 infected mice). Livers were removed and their weights recorded. Impression smears were prepared and fixed in 100\% methanol to allow determination of parasite burden and confirm infection. A small piece of liver for each animal was cut off and placed in zinc salt fixative [13] for $16 \mathrm{~h}$ and transferred to $70 \%$ ethanol for $4 \mathrm{~h}$ before processing into paraffin wax. Liver tissue samples were also immersed in $10 \%$ neutral buffered formalin solution and processed routinely into paraffin wax.

\section{Histopathology}

Four- $\mu \mathrm{m}$ tissue sections were stained with Hematoxylin and Eosin (H\&E) and analysed under light microscopy to identify lesions. The total number of granulomas together with the granuloma size (area) was calculated in a full liver section per animal using a Nikon Eclipse Ni$\mathrm{U}$ microscope and digital image analysis software (Nikon NIS Br, Nikon, Japan). Along with this, granulomas in the liver were classified into three categories according to cellular composition and evolution stage within each time point. Category 1 , corresponding to "immature granulomas", was defined as individual or few fused Kupffer cells without or with few mononuclear cells grouped loosely and harbouring a high number of amastigotes. Category 2 or "mature granulomas" were defined as tightly fused Kupffer cells surrounded by cellular infiltrate, with or without evidence of collagen deposition and with presence of some amastigotes. Category 3 or "clear granulomas" were defined as well formed granulomas with few fused Kupffer cells surrounded by a cellular infiltrate, normally with evidence of collagen deposition and without presence of amastigotes.

\section{Immunohistochemistry}

The immunohistochemical techniques are summarised in Table 1. Formalin or zinc salt fixed liver samples were cut in $4 \mu \mathrm{m}$ sections, dewaxed and rehydrated at $19{ }^{\circ} \mathrm{C}$, and placed in a fresh solution of $3 \%$ hydrogen peroxide in methanol for 15 min to block endogenous peroxidase activity. Samples were then washed in tap water. Epitope demasking was not necessary for zinc salt fixed samples. However, antigen retrieval in formalin fixed samples was achieved with enzymatic digestion, using a solution of $2 \%$ proteinase K (Dako, Glostrup, Denmark) in tris-buffered saline $(0.05 \mathrm{~mol} / \mathrm{l}$ Tris- $\mathrm{HCl} \mathrm{pH} 7.5-7.7)$ or by heat digestion (water-bath, pH 6.0 Dako buffer, $20 \mathrm{~min}, 95{ }^{\circ} \mathrm{C}$ ) 
Table 1 Antibodies and reagents used for immunohistochemistry

\begin{tabular}{|c|c|c|c|c|c|c|}
\hline Primary antibody & Antibody type & Dilution & Supplier & Epitope demasking method & Link antibody (dilution) & Buffer \\
\hline CD3 & $\begin{array}{l}\text { Rabbit vs Human CD3 } \\
\text { (polyclonal) }\end{array}$ & $1 / 500$ & $\begin{array}{l}\text { Dako (A0452) (Glostrup, } \\
\text { Denmark) }\end{array}$ & Proteinase K (Dako) & $\begin{array}{l}\text { Goat vs Rabbit } \\
(1 / 1000)\end{array}$ & TBS \\
\hline CD45R (B220) & $\begin{array}{l}\text { Rat vs Mouse CD45R } \\
\text { (B220) (monoclonal) }\end{array}$ & $1 / 100$ & $\begin{array}{l}\text { Invitrogen (RA3-6B2) (Thermo } \\
\text { Fisher, Darmstadt, Germany) }\end{array}$ & pH 6 citrate-buffer (Dako) & Goat vs Rat (1/200) & TBS \\
\hline Ly-6G (Gr1) & $\begin{array}{l}\text { Rat vs Mouse Ly-6G } \\
\text { (Gr1) (monoclonal) }\end{array}$ & $1 / 25$ & Invitrogen (RB6-8C5) & pH 6 citrate-buffer (Dako) & Goat vs Rat (1/200) & TBS \\
\hline $\mathrm{F} 4 / 80$ & $\begin{array}{l}\text { Rat vs Mouse F4/80 } \\
\text { (monoclonal) }\end{array}$ & $1 / 100$ & $\begin{array}{l}\text { BioRad (MCA 4976) } \\
\text { (Watford, Hertfordshire, UK) }\end{array}$ & None (ZSF samples) & Goat vs Rat (1/200) & TBS \\
\hline iNOS & $\begin{array}{l}\text { Rabbit vs Mouse iNOS } \\
\text { (polyclonal) }\end{array}$ & $1 / 500$ & $\begin{array}{l}\text { Merck Millipore (ABN26) } \\
\text { (Watford, Hertfordshire, UK) }\end{array}$ & None (ZSF samples) & $\begin{array}{l}\text { Goat vs Rabbit } \\
(1 / 1000)\end{array}$ & TBS \\
\hline Anti-Leish & Rabbit vs Leishmania spp. & $1 / 50$ & ISCIII (Madrid, Spain) & None & $\begin{array}{l}\text { Goat vs Rabbit } \\
(1 / 1000)\end{array}$ & TBS \\
\hline
\end{tabular}

depending on the primary antibody used (Table 1). Running tap water was then used to wash the tissue sections and the slides were then mounted onto Shandon coverplates (Thermo Fisher Scientific, Runcorn, Chesire, United Kingdom) and loaded into Sequenza ${ }^{\odot}$ trays (Shandon Scientific). Once mounted, the slides were washed with tris-buffered saline (TBS) $(0.85 \% \mathrm{NaCl}$, $0.0605 \%$ Tris, adjusted to $\mathrm{pH} 7.5$ using $1 \mathrm{M} \mathrm{HCl}$ ) and then $190 \mu \mathrm{l}$ of Universal Blocker ${ }^{\mathrm{Ts}}$ Blocking Buffer in TBS (Thermo Fisher Scientific, Runcorn, Chesire, United Kingdom) was added as blocking agent. After $20 \mathrm{~min}, 190 \mu \mathrm{l}$ of the primary antibody was added. The incubation time varied for each primary antibody used. After two washes with TBS buffer, $190 \mu$ l of biotinylated link antibody (Table 1) and link block were added, followed by two further buffer washes, $30 \mathrm{~min}$ later. Primary and secondary antibody binding was amplified using Ultra-Sensitive ABC Peroxidase Rabbit IgG Staining Kit (Thermo Fisher Scientific, Runcorn, Chesire, United Kingdom) and visualized using the Vector ${ }^{\odot}$ NovaRED $^{\text {rM }}$ Substrate Kit (Vector Laboratories, Burlingame, California, USA). Unbound conjugate was removed prior to Vector $^{\ominus}$ NovaRED $^{\text {тx }}$ Substrate Kit application with two buffer washes. Slides were then washed in purified water, removed from the coverplates and placed in a rack. Samples were rinsed with tap water for $5 \mathrm{~min}$, before being placed in Mayer's Haematoxylin counterstain, followed by a further wash in tap water. Finally, sections were dehydrated, cleared and mounted for analysis.

\section{Image analysis}

Immunolabelled sections were analysed using light microscopy and digital image analysis (Nikon NIS Br, Nikon Instruments Europe BV, Amsterdam, The Netherlands). The slides were examined with the $40 \times$ objective to give a final magnification of $400 \times$, to ascertain the percentage of immunostained area in the lesion. The whole area of the granuloma was selected as region of interest (ROI), and the area with immunohistochemically positive reaction within the ROI was calculated by the software after setting the thresholds. The results are expressed as the percentage of positively immunolabelled area within the total area of the granuloma.

\section{Statistics}

Chi-square test was applied to analyse differences between the number and category of granulomas per time point in the liver. Student's t-test was used to assess the significance of the differences in the size between granuloma categories.

For IHC, Student's t-test was performed to compare the mean expression of each immune marker within granuloma categories and time points. Differences were considered significant at $P<0.05$. The results of immunohistochemical analyses are expressed as group mean \pm standard deviation (SD). All analyses were conducted using SPSS 19 software package (SPSS Inc., Chicago, IL, 60606, USA) and GraphPad Prism 7.0 (San Diego, USA).

\section{Results \\ Confirmation of infection}

The hepatic parasite burden $15 \mathrm{dpi}$, expressed in Leishman-Donovan units (LDUs), was $456 \pm 65$ (group mean \pm SD), confirming previously observed levels of infection. Liver weights (group mean \pm SD) at $15 \mathrm{dpi}, 35$ dpi and $63 \mathrm{dpi}$ were $1041 \pm 90 \mathrm{mg}, 1365 \pm 107 \mathrm{mg}$ and $1412 \pm 71 \mathrm{mg}$, respectively.

\section{Histopathology}

Granulomas and aggregations of macrophages were present in the livers of all infected animals from $15 \mathrm{dpi}$ onwards (Fig. 1). After the analysis of an entire section of liver for each animal, we characterized (categories) and measured a total of 2467 granulomas from the infected animals. Statistically significant differences 

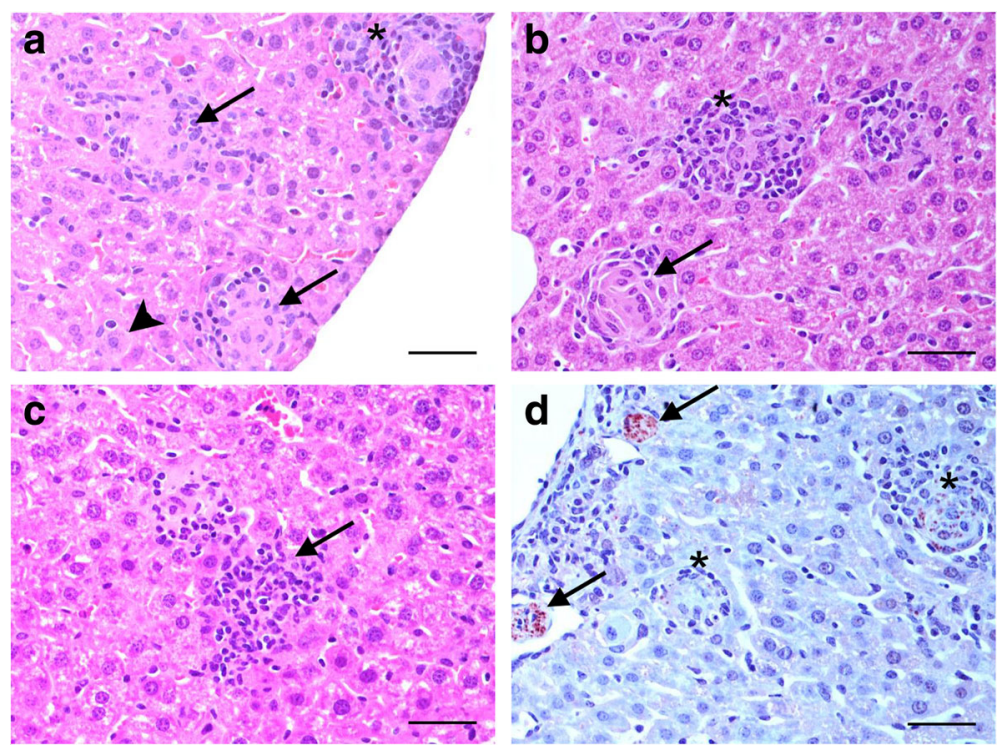

Fig. 1 a H\&E-stained liver section 15 dpi (400x). Kupffer cell harbouring a high amount of amastigotes (arrowhead). Immature granulomas formed by fused macrophages harbouring few amastigotes (arrows). Well-formed mature granuloma with a complete cellular infiltrate $\left(^{*}\right)$. b H\&E-stained liver section 35 dpi (400x). Mature functional granuloma with few fused macrophages harbouring very few amastigotes (*). Clear granuloma without amastigotes and with presence of collagen deposition (arrow). c H\&E-stained liver section 63 dpi (400x). Clear granuloma composed mainly of lymphocytes (arrow). d Immunohistochemical detection of Leishmania donovani antigens (400x). Immature granulomas harbouring a high amount of amastigotes (arrows) and mature granulomas with fewer number of amastigotes $(*)$. Scale-bars: $100 \mu \mathrm{m}$

$(P<0.001)$ were found in the mean size of granulomas, depending on their category (Table 2). Mature granulomas were larger compared to the other two categories (Immature $v s$ mature: $t=-38.410, P<0.001$; Immature vs clear: $t=4.181, P<0.001$ and mature $v s$ clear: $t=$ $18.669, P<0.001)$. The granuloma size of each category remained homogeneous between the different time points analysed.

The number and category of hepatic granulomas varied among the three time points (Fig. 2). At 15 dpi, 75\% of granulomas were immature, with no clear granulomas being observed. At $35 \mathrm{dpi}$, the total number of granulomas increased by $40 \%$ compared to $15 \mathrm{dpi}$. In this case, $57 \%$ of granulomas were mature and almost $4 \%$ had begun to be resolved (clear granulomas). At $63 \mathrm{dpi}$, the total number of granulomas was reduced by $30 \%$ in comparison with $35 \mathrm{dpi}$, with $62 \%$ of mature granulomas and almost $17 \%$ of clear granulomas.

Table 2 Mean size of granulomas per category

\begin{tabular}{lllll}
\hline Granuloma category & Mean size $\left(\mu \mathrm{m}^{2}\right)$ & Number $(n)$ & SD & Error \\
\hline Immature & $30,434.05^{*}$ & 1061 & $13,471.478$ & 413.578 \\
Mature & $66,612.02^{*}$ & 1238 & $28,030.675$ & 796.660 \\
Clear & $25,925.49^{*}$ & 168 & 9323.050 & 719.289 \\
\hline
\end{tabular}

Abbreviation: $S D$ standard deviation

${ }^{*} P<0.001$, when mean size is compared among the three granuloma categories

\section{Immunohistochemistry \\ Detection of Leishmania spp. antigen}

A specific and strong positive signal was observed for amastigotes inside Kupffer cells in the liver (Fig. 1d).

\section{Kupffer cells (F4/80+) and iNOS+ cells}

Immunohistochemistry (IHC) for F4/80 was used to locate Kupffer cells in the liver. A decrease in the expression of F4/80 was observed as the granuloma category evolved within the same time point. However, a statistically significant increase in the expression of F4/80 was noted when immature and mature granulomas were compared across the different time points (15 vs 35 dpi, immature: $t=-3.291, P<0.001$ and mature: $t=-2.202, P<0.029 ; 15$ vs $63 \mathrm{dpi}$, immature: $t=-5.691, P<0.001$ and mature: $t=-4.335, P<0.001$ ) There were no differences in the expression of F4/80 observed for clear granulomas when animals euthanized at 35 and 63 dpi were compared. The highest expression of $\mathrm{F} 4 / 80$ was found in immature granulomas, regardless of the time point analyzed (Fig. 3). Distribution of F4/80+ cells within granulomas was characterized by a strong signal in Kupffer cells situated at the periphery of granulomas and a lighter signal in fused Kupffer cells containing $L$. donovani amastigotes in the centre of the granuloma (Fig. 3).

Immunohistochemical staining for iNOS also showed a higher mean percentage staining in immature granulomas, regardless of the time point. As occurred 


\section{Hepatic granulom as}

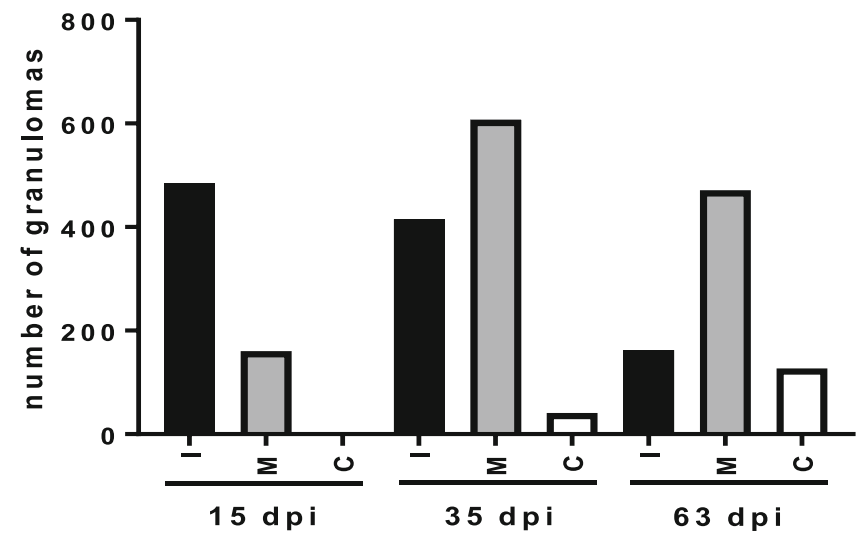

\begin{tabular}{|c|c|c|c|c|}
\hline & \multicolumn{3}{|c|}{ Granuloma category } & \\
\hline Time point & Immature & Mature & Clear & Total \\
\hline $15 \mathrm{dpi}$ & $484(75 \%)$ & $160(25 \%)$ & & $644(100 \%)$ \\
\hline $35 \mathrm{dpi}$ & $415(39 \%)$ & $607(57 \%)$ & $41(4 \%)$ & $1063(100 \%)$ \\
\hline $63 \mathrm{dpi}$ & $162(21 \%)$ & $471(62 \%)$ & $127(17 \%)$ & $760(100 \%)$ \\
\hline Total & $\mathbf{1 0 6 1}$ & $\mathbf{1 2 3 8}$ & $\mathbf{1 6 8}$ & $\mathbf{2 4 6 7}$ \\
\hline
\end{tabular}

Fig. 2 Distribution (total numbers and percentages) of hepatic granulomas induced by Leishmania donovani divided into category 1 (immature), category 2 (mature) and category 3 (clear) at three different time points (15 dpi, 35 dpi and 63 dpi)

for $\mathrm{F} 4 / 80+$ immunostaining, there was a decrease in the expression of iNOS as the granuloma categories evolved within the same time point. However, compared with $\mathrm{F} 4 / 80+$ cells, the highest expression of iNOS was observed at $35 \mathrm{dpi}$ and not at $63 \mathrm{dpi}$. In fact, there was a statistically significant reduction in the expression of iNOS for the three granuloma categories at $63 \mathrm{dpi}$, when compared to $35 \mathrm{dpi}$ (immature: $t=4.589$, $P<0.001$; mature: $t=10.520, P<0.001$; clear: $t=8.653$, $P<0.001$ ) (Fig. 3). Positive staining for iNOS was

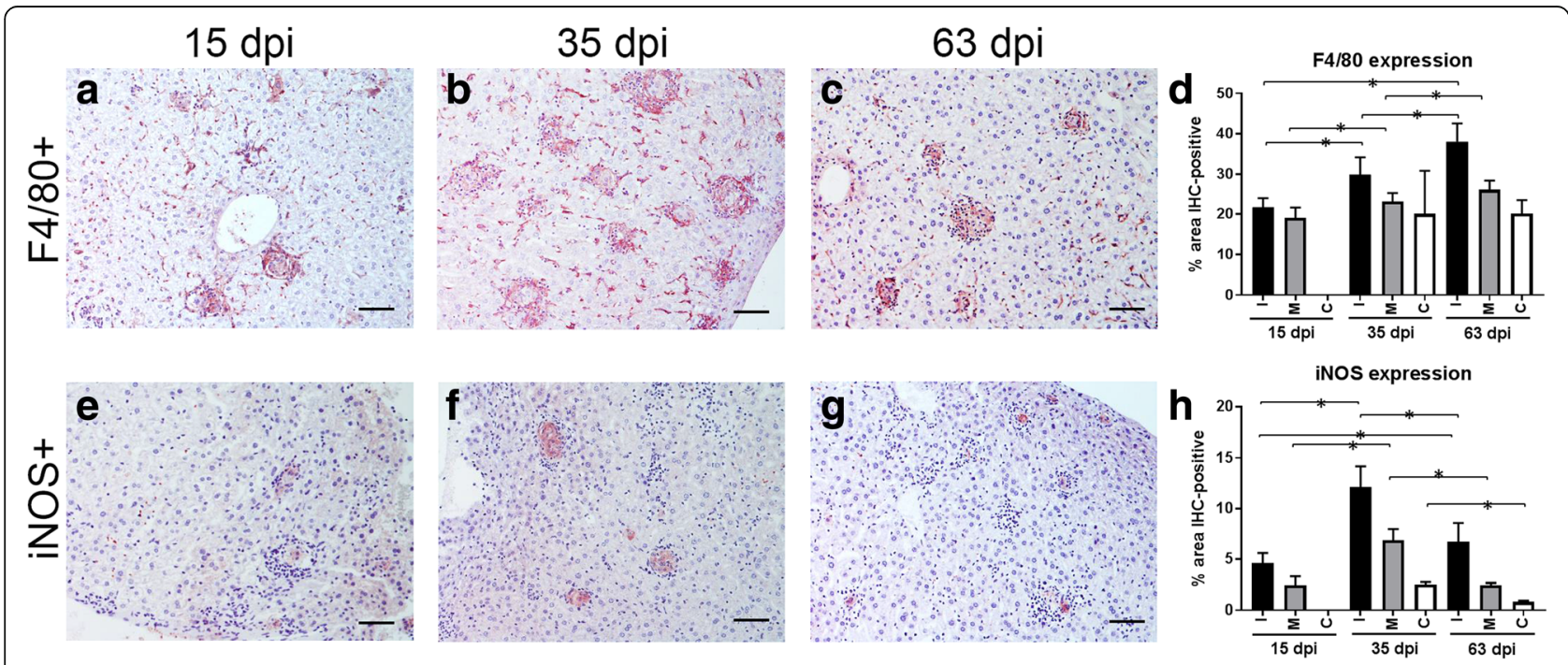

Fig. 3 Immunohistochemical detection of macrophages (F4/80+) within hepatic granulomas at 15 dpi (a), 35 dpi (b) and 63 dpi (c). d Percentage of immunostained area for $\mathrm{F} 4 / 80$ at 15, 35 and 63 dpi within immature (I), mature (M) and clear (C) granulomas. Immunohistochemical detection of iNOS+ cells at $15 \mathrm{dpi}(\mathbf{e}), 35 \mathrm{dpi}(\mathbf{f})$ and $63 \mathrm{dpi}(\mathbf{g})$. $\mathbf{h}$ Percentage of immunostained area for iNOS at 15, 35 and $63 \mathrm{dpi}$ within immature (I), mature $(\mathrm{M})$ and clear $(\mathrm{C})$ granulomas. ${ }^{*} P<0.05$. Scale-bars: $50 \mu \mathrm{m}$ 
mainly observed in the cytoplasm of fused Kupffer cells in the centre of the granuloma and was especially marked in immature granulomas (Fig. 3).

\section{$T$ lymphocytes (CD3+)}

An increase in the expression of CD3 was noted as granuloma categories evolved in animals at 35 and 63 $\mathrm{dpi}$, while a reduction was observed at $15 \mathrm{dpi}$. The highest expression of CD3 was observed in animals euthanized at $63 \mathrm{dpi}$. The only statistically significant differences were observed when comparing mature granulomas between animals euthanized at 35 and 63 dpi $(t=-2.071, P<0.001)$ and immature granulomas at 15 and $35 \mathrm{dpi}(t=3.507, P<0.001)$ (Fig. 4). Distribution of $\mathrm{CD} 3+$ cells was similar within granuloma categories and time points. CD3+ cells were dispersed in the cellular infiltrate surrounding the fused Kupffer cells situated in the granuloma centre (Fig. 4).

\section{B lymphocytes (CD45R-B220+)}

Immunohistochemical staining for CD45R-B220 showed the location of $\mathrm{B}$ lymphocytes within the granuloma. An increase in the expression of CD45R-B220 was observed as granuloma categories evolved within the same time point (Fig. 4). When comparing granulomas from mice euthanized at 15 and $35 \mathrm{dpi}$, a statistically significant reduction in the expression of CD45RB220 was observed for immature granulomas $(t=2.029$, $P<0.043)$, while a statistically significant increase was observed for mature granulomas $(t=-2.213, P<0.027)$. The expression of CD45R-B220 in mature granulomas was quite homogeneous when comparing mice euthanized at 35 and 63 dpi (Fig. 4). Most of the cells shaping the cellular infiltrate surrounding the fused Kupffer cells were CD45R-B220+ cells when compared with CD3+ cells (Fig. 4).

\section{Neutrophils (Ly-6G+)}

The expression of Ly-6G within granulomas was higher in mature compared to immature and clear granulomas. Within mature granulomas a significantly higher expression of Ly-6G was observed at $15 \mathrm{dpi}$ when compared to $35 \mathrm{dpi}(t=3.019, P<0.043)$ and 63 dpi $(t=4.034, P<0.035)$. In clear granulomas, neutrophils were only observed occasionally. The expression of Ly-6G was generally lower compared to the other studied cell markers, particularly at later time points (Fig. 4).

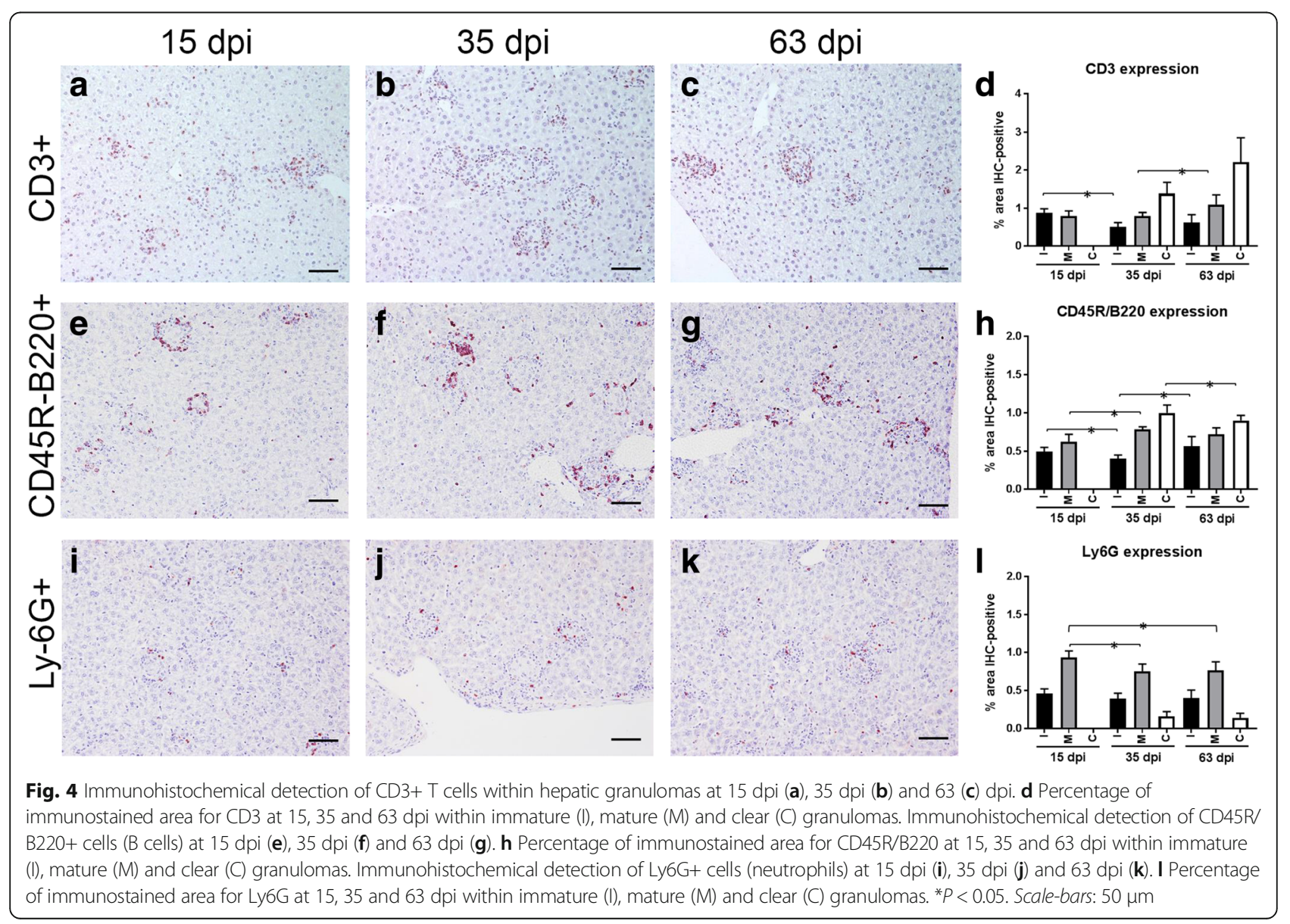




\section{Discussion}

The histopathological hallmark of hepatic resistance to visceralizing species of Leishmania is the development of functional granulomas $[10,11,14]$. Here we used IHC as a tool to characterise the different cells involved in the hepatic granuloma development at different stages of experimental VL in BALB/c mice, complemented by quantitative image analysis and detection of Leishmania antigen.

Immunohistochemical methods described here are a powerful tool to characterise host responses to infection in situ in mouse models of Leishmania spp. infection. These have also been used in other granulomatous diseases that share some, though not all properties with VL [15-18]. Additionally these techniques can be utilised to evaluate the cellular mechanism of new vaccines, drugs and treatment regimens [19-21]. Therefore this study provides in depth insights into the cellular dynamics of experimental VL in a context relevant to advancing health.

In agreement with previous studies [22, 23], we observed that the majority of hepatic granulomas at $15 \mathrm{dpi}$ were immature, with a heavy presence of amastigotes. Early amastigote replication in tissue macrophages is regulated by the phagosomal proton-cation antiporter encoded by the Slc11a1 gene (formerly Nramp1), and high amastigote loads in the livers of BALB/c mice have been linked to mutations in the Slc11a1 gene [24-27]. Control of hepatic parasite growth at later stages of infection corresponds to the development of acquired immune mechanisms $[27,28]$. The increase in the percentage of mature granulomas and the appearance of clear granulomas at 35 and 63 dpi reflect the onset of acquired immune responses and disease control, as the leishmanicidal efficacy of hepatic granulomas is dependent on their degree of maturation [23, 29]. In this sense, it has been proposed that determining the degree of maturation of hepatic granulomas constitutes an effective tool for selecting VL vaccine candidates [30].

We also observed significant differences in hepatic granuloma sizes between categories, possibly linked to differences in the cellular composition of respective granulomas in each category. Immature granulomas are shaped by the presence of phagocytic cells, whereas mature granulomas contain higher numbers of lymphocytes, as observed by immunohistochemical detection of $\mathrm{T}$ and $\mathrm{B}$ cells. Clear granulomas are smaller than mature granulomas and contain less F4/80+ cells.

The highest expression of F4/80 found in immature granulomas and the decrease in the expression of F4/80 observed as the granuloma category evolved within the same time point, reflect that resident macrophages are the first line of defence against Leishmania parasites within this tissue. Moreover, monocytes are recruited into the granuloma by chemokines (CCL3, CCL2 and CXCL10) secreted by Kupffer cells infected with the parasite [27].
Similarly to F4/80+ cells, immunohistochemical staining for iNOS showed a higher mean percentage staining in immature granulomas regardless of the time point, and there was a decrease in the expression of iNOS as the granuloma categories evolved within the same time point. Hepatic resistance against $L$. donovani infection correlates well with the generation of reactive oxygen and reactive nitrogen intermediates [12]. Macrophages are the main producers of iNOS, especially when activated by the intervention of $\mathrm{T}$ lymphocytes. Nitric oxide produced by iNOS is believed to be of prime importance in the cure of murine leishmaniasis, and in activating murine macrophages to kill intracellular parasites [31]. This has been illustrated by studies of iNOS gene knockout mice, which do not self-resolve infection [32]. The statistically significant reduction in the expression of iNOS for the three granuloma categories at $63 \mathrm{dpi}$, when compared to $35 \mathrm{dpi}$, may be related to the presence of lower numbers of Leishmania amastigotes in the liver at this phase of infection. The course of infection in BALB/ c mice infected with the $L$. donovani strain used here is well documented [33, 34]. An increase in hepatic parasite burden is observed until amastigote growth is controlled around $28 \mathrm{dpi}$, followed by clearance of parasites at later time points. In our study, complete absence of Leishmania amastigotes was not observed by $63 \mathrm{dpi}$. It has previously been reported that sterile immunity in the liver is not achieved, but the presence of a residual parasite population is thought to incite a small but enduring immune response that provides long-term immunity to reinfection [27].

Expression of CD3 was lower at $15 \mathrm{dpi}$ and increased as granuloma categories evolved at 35 and 63 dpi. The highest expression of CD3 was observed at the last time point, which is in agreement with the continuous maturation of hepatic granulomas. Gene knockout and antibody neutralization studies showed that granuloma maturation in the liver of infected mice requires CD4 and CD8 $\mathrm{T}$ cells and proinflammatory cytokines such as IL-12, IFN-g and IL-2 to be functional [29, 35, 36].

Most of the cells that shaped the cellular infiltrate surrounding the fused Kupffer cells were CD45R-B220+ cells and the expression of this marker increased as the granuloma categories evolved within each time point. B cells have previously been observed in hepatic granulomas of $L$. donovani infected BALB/c mice [35] and have been shown to accumulate in hepatic granulomas in infected mice over time, exhibiting a highly motile behaviour [37]. They play a role in preventing liver pathology via control of neutrophil infiltration [38]. The increase of CD45R-B220+ cells over time is also related to the establishment of acquired immunity during the maturation process of granulomas [14]. 
The number of Ly6G+ neutrophils within the hepatic granulomas was low and this cell population was mainly observed in mature granulomas at all time points. Previous studies have demonstrated protective effects of neutrophils in livers of $L$. donovani infected mice and small numbers of infiltrating neutrophils have been observed in granulomas at $14 \mathrm{dpi}[38,39]$. A contribution of neutrophils to the maturation of functional hepatic granulomas and induction of hepatic iNOS has been suggested [39].

\section{Conclusions}

We observed that hepatic lesions start as a basic fusion of Kupffer cells, producing high levels of iNOS, and move to a more complex granuloma, related to an adaptive immune response with the presence of higher numbers of $\mathrm{B}$ and $\mathrm{T}$ lymphocytes and a final resolution towards collagen deposition. The analyses described here, based on infection with an established laboratory strain of $L$. donovani, can be applied to other hostparasite combinations, including genetically manipulated parasites and recent clinical isolates. The panel of cellular markers can be extended to include other, lessstudied cell types. Paraffin blocks from this study have been archived and are available for future analysis.

\section{Abbreviations}

CL: Cutaneous leishmaniasis; dpi: Days post-infection; H\&E: Hematoxylin and eosin; IHC: Immunohistochemistry; iNOS: Inducible nitric oxide synthase; LDU: Leishman-Donovan units; NTD: Neglected tropical disease; ROI: Region of interest; SD: Standard deviation; VL: Visceral leishmaniasis

\section{Acknowledgements}

We are grateful to Dr Mercedes Dominguez, from ISCIII, Madrid, Spain, for kindly providing the anti-Leishmania antibody used in this study.

\section{Funding}

The generation of tissues analysed in this study was supported by a grant jointly funded by the UK Medical Research Council and the UK Department for International Development under the MRC/DFID Concordat agreement (grant MR/J008702/1). Additional funding was received from the Immunology and Infection Department at LSHTM. WLGJ was supported by Junta de Extremadura (Spain) and European Social Fund (grant \#PO14022). IL was supported by the Science without Borders Programme (CAPES and CNPq, Brazil). The digital pathology workstation used in this study was funded by vHive (Veterinary Health Innovation Engine) at the University of Surrey. The funders had no role in study design, data collection and interpretation, or the decision to submit the work for publication.

\section{Availability of data and materials}

The data supporting the conclusions of this article are included within the article. The datasets used and/or analysed are available from the corresponding author upon reasonable request.

\section{Authors' contributions}

FJS and KS designed the study and performed the animal experiments and post-mortem examinations. WLGJ and IL carried out the immunohistochemical analysis and image analyses. FJS, KS and WLGJ wrote the manuscript. All authors read and approved the final manuscript.

\section{Ethics approval}

All animal experiments were approved by the Animal Welfare and Ethics Review Board at LSHTM and performed under license in accordance with the Animals (Scientific Procedures) Act of 1986 (UK Home Office project license PPL70/8207).
Consent for publication

Not applicable.

\section{Competing interests}

The authors declare that they have no competing interests.

\section{Publisher's Note}

Springer Nature remains neutral with regard to jurisdictional claims in published maps and institutional affiliations.

\section{Author details}

${ }^{1}$ Department of Pathology and Infectious Diseases, School of Veterinary Medicine, University of Surrey, Guildford, UK. ²Fundação Oswaldo Cruz, Centro de Pesquisas Gonçalo Moniz, Salvador, Bahia, Brazil. ${ }^{3}$ Faculty of Infectious and Tropical Diseases, London School of Hygiene \& Tropical Medicine, London, UK.

Received: 13 March 2017 Accepted: 8 January 2018

Published online: 31 January 2018

\section{References}

1. Alvar J, Vélez ID, Bern C, Herrero M, Desjeux P, Cano J, et al. Leishmaniasis worldwide and global estimates of its incidence. PLoS One. 2012;7:e35671.

2. Ready PD. Epidemiology of visceral leishmaniasis. Clin Epidemiol. 2014;6: $147-54$

3. Reithinger R, Dujardin JC, Luozir H, Pirmez C, Alexander B, Brooker S. Cutaneous leishmaniasis. Lancet Infect Dis. 2007:7:581-96.

4. Chappius F, Sundar S, Hailu A, Ghalib H, Rijal S, Peeling RW, et al. Visceral leishmaniasis: what are the needs for diagnosis, treatment and control? Nat Rev Microbiol. 2007:5:873-82

5. Pigott DM, Bhatt S, Golding N, Duda KA, Battle KE, Brady OJ, et al. Global distribution maps of the leishmaniases. Elife. 2014;3:e02851.

6. Laurenti MD, Rossi CN, Matta VLRD, Tomokane TY, Corbett CEP, Secundino $\mathrm{NFC}$, et al. Asymptomatic dogs are highly competent to transmit Leishmania (Leishmania) infantum chagasi to the natural vector. Vet Parasitol. 2013:196:296-300.

7. Maroli M, Rossi L, Baldelli R, Capelli G, Ferroglio E, Genchi C, et al. The northward spread of leishmaniasis in Italy: evidence from retrospective and ongoing studies on the canine reservoir and phlebotomine vectors. Tropical Med Int Health. 2008;13:256-64.

8. Seifert K. Structures, targets and recent approaches in anti-leishmanial drug discovery and development. Open Med Chem J. 2011;5:31-9.

9. Croft SL, Olliaro P. Leishmaniasis chemotherapy - challenges and opportunities. Clin Microbiol Infect. 2011;17:1478-83.

10. Kaye PM, Beattie L. Lessons from other diseases: granulomatous inflammation in leishmaniasis. Semin Immunopathol. 2016;3:249-60.

11. Murray HW. Tissue granuloma structure-function in experimental visceral leishmaniasis. Int J Exp Pathol. 2001;82:249-67.

12. Kaye PM, Svensson M, Ato M, Maroof A, Polley R, Stager S, et al. The immunopathology of experimental visceral leishmaniasis. Immunol Rev. 2004;201:239-53.

13. Hicks DJ, Johnson L, Mitchell SM, Gough J, Cooley WA, La Ragione RM, et al. Evaluation of zinc salt based fixatives for preserving antigenic determinants for immunohistochemical demonstration of murine immune system cell markers. Biotech Histochem. 2006;81:23-30.

14. Moore JW, Moyo D, Beattie L, Andrews PS, Timmis J, Kaye PM. Functional complexity of the Leishmania granuloma and the potential of in silico modeling. Front Immunol. 2013:4:35.

15. Stefanaki K, Tsivitanidou-Kakourou T, Stefanaki C, Valari M, Argyrakos T, Konstantinidou CV, et al. Histological and immunohistochemical study of granuloma annulare and subcutaneous granuloma annulare in children. J Cutan Pathol. 2007;34:392-6.

16. Janssen CE, Rose CD, De Hertogh G, Martin TM, Bader Meunier B, Cimaz R, et al. Morphologic and immunohistochemical characterization of granulomas in the nucleotide oligomerization domain 2-related disorders Blau syndrome and Crohn disease. J Allergy Clin Immunol. 2012;129:1076-84.

17. Aranday-Cortes E, Bull NC, Villarreal-Ramos B, Gough J, Hicks D, Ortiz-Pelaez A, et al. Upregulation of IL-17A, CXCL9 and CXCL10 in early-stage granulomas induced by Mycobacterium bovis in cattle. Transbound Emerg Dis. 2013;60:525-37. 
18. Salguero FJ, Gibson S, Garcia-Jimenez W, Gough J, Strickland TS, Vordermeier HM, et al. Differential cell composition and cytokine expression within lymph node granulomas from BCG-vaccinated and non-vaccinated cattle experimentally infected with Mycobacterium bovis. Transbound Emerg Dis. 2017;64:1734-49.

19. Johnson L, Gough J, Spencer Y, Hewinson G, Vordermeier M, Wangoo A. Immunohistochemical markers augment evaluation of vaccine efficacy and disease severity in bacillus Calmette-Guerin (BCG) vaccinated cattle challenged with Mycobacterium bovis. Vet Immunol Immunopathol. 2006; 111:219-29.

20. Daneshvar H, Sedghy F, Dabiri S, Kamiabi H, Molaei MM, Phillips S, Burchmore R. Alteration in mononuclear cell subpopulations in dogs immunized with gentamicin-attenuated Leishmania infantum. Parasitology. 2012;139:1689-96

21. Seifert K, Juhls C, Salguero FJ, Croft SL. Sequential chemoimmunotherapy of experimental visceral leishmaniasis using a single low dose of liposomal amphotericin B and a novel DNA vaccine candidate. Antimicrob Agents Chemother. 2015;59:5819-23.

22. Gutierrez Y, Maksem JA, Reiner NE. Pathologic changes in murine leishmaniasis (Leishmania donovani) with special reference to the dynamics of granuloma formation in the liver. Am J Pathol. 1984;114:222-30.

23. Carrión J, Nieto A, Iborra S, Iniesta V, Soto M, Folqueira C, et al. Immunohistological features of visceral leishmaniasis in BALB/C mice. Parasite Immunol. 2006;28:173-83.

24. Crocker PR, Blackwell JM, Bradley DJ. Expression of the natural resistance gene Lsh in resident liver macrophages. Infect Immun. 1984:43:1033-40.

25. Leclercq V, Lebastard M, Belkaid Y, Louis J, Milon G. The outcome of the parasitic process initiated by Leishmania infantum in laboratory mice: a tissue-dependent pattern controlled by the Lsh and MHC loci. J Immunol. 1996;157:4537-45.

26. Blackwell JM, Goswami T, Evans CAW, Sibthorpe D, Papo N, White JK, et al, SLC11A1 (formerly NRAMP1) and disease resistance. Cell Microbiol. 2001;3: 773-84.

27. Stanley AC, Engwerda CR. Balancing immunity and pathology in visceral leishmaniasis. Immunol Cell Biol. 2007:85:138-47.

28. Lipoldova M, Demant P. Genetic susceptibility to infectious disease: lessons from mouse models of leishmaniasis. Nat Rev Genet. 2006;7:294-305.

29. Murray HW, Squires KE, Miralles CD, Stoeckle MY, Granger AM, Granelli-Piperno A, et al. Acquired resistance and granuloma formation in experimental visceral leishmaniasis. Differential T cell and lymphokine roles in initial versus established immunity. J Immunol. 1992;148:1858-63.

30. Nieto A, Domínguez-Bernal G, Orden JA, De La Fuente R, Madrid-Elena N, Carrión J. Mechanisms of resistance and susceptibility to experimental visceral leishmaniosis: BALB/c mouse versus Syrian hamster model. Vet Res. 2011;42:39.

31. Liew FY, Wei XQ, Proudfoot L. Cytokines and nitric oxide as effector molecules against parasitic infections. Philos Trans R Soc Lond Ser B Biol Sci. 1997:352:1311-5

32. Murray HW, Nathan F. Macrophage microbicidal mechanisms in vivo: reactive nitrogen versus oxygen intermediates in the killing of intracellular visceral Leishmania donovani. J Exp Med. 1999;189:741-6.

33. Engwerda CR, Ato M, Kaye PM. Macrophages, pathology and parasite persistence in experimental visceral leishmaniasis. Trends Parasitol. 2004;20:524-30.

34. Riede O, Seifert K, Oswald D, Endmann A, Hock C, Winkler A, et al. Preclinical safety and tolerability of a repeatedly administered human leishmaniasis DNA vaccine. Gene Ther. 2015;22:628-35.

35. McElrath MJ, Murray HW, Cohn ZA. The dynamics of granuloma formation in experimental visceral leishmaniasis. J Exp Med. 1988;167:1927-37.

36. Squires KE, Schreiber RD, McElrath MJ, Rubin BY, Anderson SL, Murray HW. Experimental visceral leishmaniasis: role of endogenous IFN-gamma in host defense and tissue granulomatous response. J Immunol. 1989:143:424-9.

37. Moore JW, Beattie L, Dalton JE, Owens BMJ, Maroof A, Coles MC, et al. B cell: T cell interactions occur within hepatic granulomas during experimental visceral leishmaniasis. PLoS One. 2012;7:e34143.

38. Smelt SC, Cotterell SE, Engwerda CR, Kaye PM. B cell-deficient mice are highly resistant to Leishmania donovani infection, but develop neutrophilmediated tissue pathology. J Immunol. 2000;164:3681-8.

39. McFarlane E, Perez C, Charmoy M, Allenbach C, Carter KC, Alexander J, et al. Neutrophils contribute to development of a protective immune response during onset of infection with Leishmania donovani. Infect Immun. 2008;76:532-41.

\section{Submit your next manuscript to BioMed Central and we will help you at every step:}

- We accept pre-submission inquiries

- Our selector tool helps you to find the most relevant journal

- We provide round the clock customer support

- Convenient online submission

- Thorough peer review

- Inclusion in PubMed and all major indexing services

- Maximum visibility for your research

Submit your manuscript at www.biomedcentral.com/submit 